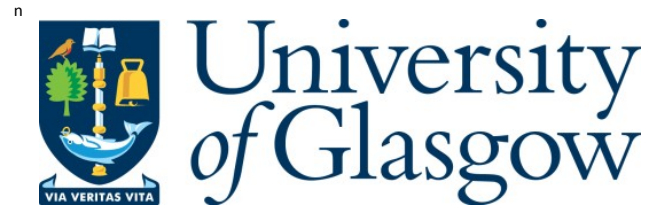

Qiu, Z., Qiu, Y ., Demore, C. E.M ., and Cochran, S. (2016) Implementation of a PM N-PT piezocrystal-based focused array with geodesic faceted structure.

Ultrasonics, 69, pp. 137-143.

There may be differences between this version and the published version. $Y$ ou are advised to consult the publisher's version if you wish to cite from it.

http://eprints.gla.ac.uk/118495/

Deposited on: 18 A pril 2016

Enlighten - Research publications by members of the U niversity of Glasgow http://eprints.gla.ac.uk 


\title{
Implementation of a PMN-PT Piezocrystal-based Focused Array with Geodesic Faceted Structure
}

\author{
Zhen Qiu ${ }^{1}$, Yongqiang Qiu ${ }^{2}$, Christine E. M. Demore ${ }^{2}$, Sandy Cochran ${ }^{2}$ \\ ${ }^{1}$ Department of Electronics and Electrical Engineering, University of Strathclyde, Glasgow, UK \\ ${ }^{2}$ School of Engineering, University of Glasgow, Glasgow, UK
}

\begin{abstract}
The higher performance of relaxor-based piezocrystals compared with piezoceramics is now well established, notably including improved gain-bandwidth product, and these materials have been adopted widely for biomedical ultrasound imaging. However, their use in other applications, for example as a source of focused ultrasound for targeted drug delivery, is hindered in several ways. One of the issues, which we consider here, is in shaping the material into the spherical geometries used widely in focused ultrasound. Unlike isotropic unpoled piezoceramics that can be shaped into a monolithic bowl then poled through the thickness, the anisotropic structure of piezocrystals make it impossible to machine the bulk crystalline material into a bowl without sacrificing performance. Instead, we report a novel faceted array, inspired by the geodesic dome structure in architecture, which utilizes flat piezocrystal material and maximizes fill factor. Aided by $3 \mathrm{D}$ printing, a prototype with $\mathrm{f} \# \approx 1.2$, containing 96 individually addressable elements was manufactured using 1-3 connectivity PMN-PT piezocrystal - epoxy composite. The fabrication process is presented and the array was connected to a 32-channel controller to shape and steer the beam for preliminary performance demonstration. At an operating frequency of $1 \mathrm{MHz}$, a focusing gain around 30 was achieved and the side lobe intensities were all at levels below $-12 \mathrm{~dB}$ compared to main beam. We conclude that, by taking advantage of contemporary fabrication techniques and driving instrumentation, the geodesic array configuration is suitable for focused ultrasound devices made with piezocrystal.
\end{abstract}

Keywords - Geodesic array; PMN-PT piezocrystal; focused ultrasound; 3D printing

\section{Introduction}

The use of focused ultrasound as a means to deliver energy has been expanding since the 1990s in areas including high intensity focused ultrasound surgery (FUS) [1], ultrasound-mediated targeted drug delivery (UmTDD) [2] and particle trapping and manipulation [3]. The focused ultrasound beam can be achieved geometrically with a curved piezoelectric bowl or acoustic lens or electrically with a multi-element phased array. The combination of both approaches is also common, with many arrays with overall concave geometry for focusing used in research and clinical practice [4-6]. This is because concave arrays can provide higher focal intensities than planar arrays with similar overall dimensions. Furthermore, when only a limited range of 
movement in the focal point is necessary, fewer elements and simpler time delays are needed, reducing the complexity of the driving circuitry.

Historically, piezoceramics have been the most commonly used materials in ultrasound transducer arrays but, recently, the improved performance offered by relaxor-based piezocrystals in the $\mathrm{Pb}\left(\mathrm{Mg}_{1 / 3} \mathrm{Nb}_{2 / 3}\right) \mathrm{O}_{3}$ $\mathrm{PbTiO}_{3}$ (PMN-PT) family has been recognized. Piezocrystals are now used widely in biomedical ultrasound imaging and investigation of their possibilities is under way in nondestructive evaluation, particle manipulation and underwater sonar [7-10] based on piezoelectric properties including $k_{33} \approx 90 \%$ and $d_{33}>1500 \mathrm{pC} / \mathrm{N}$ [11]. Bulk, monolithic piezocrystal has little advantage over piezoceramic, with the values of $k_{t}$ being similar, but improved performance can be realized in configurations such as the planks used in biomedical imaging arrays and the pillars used in piezocomposites.

PMN-PT itself, termed a Generation I material, is susceptible to changes in functional properties at elevated temperature and pressure [12], but Generations II and III, respectively exemplified by ternary PINPMN-PT and doped ternary Mn:PIN-PMN-PT, are under development to reduce these effects whilst maintaining much of the performance advantage over piezoceramic, e.g. in terms of $k_{33}$ and $d_{33}$ that determine piezocomposite performance. Piezocrystal composites thus have potential to improve the effectiveness of focused ultrasound transducers and the work reported here contributes to this by describing the fabrication and testing of a 2D concave phased array made with Generation I piezocrystal.

A widely-reported method to fabricate concave transducer arrays is to place individual single-element transducers in a prefabricated frame at predetermined positions $[6,13]$. This requires manufacture of each individual array element separately and the presence of the frame can significantly reduce the array fill-factor, i.e. the percentage surface coverage of the active piezoelectric material, and thus the focusing gain and acoustic energy output of the transducer. As an alternative, Raju et al. [14] proposed an array design using a spherically-focused ceramic bowl with printed electrodes defining the elements. However, fabrication of curved sections from piezocrystal boules would be wasteful of material which costs $\$ 0.5-3 / \mathrm{mm}^{3}$, and more importantly, performance would be decreased by the lack of alignment between the piezocrystal axes and the surface of the array. Mechanical forming to focus piezocrystal transducers has been achieved successfully using mechanical hard-press [15] and dimpling techniques [16], but reports are limited to single-element transducers. Other possibilities are to thermally form a 1-3 connectivity piezoelectric - polymer composite into the desired shape [17] or to use flexible polymers for flexible composites [18], but these may be difficult at the 
frequencies of interest here, below 1.0 MHz, and the values of volume fraction, e.g. $V F>50 \%$, used in focused ultrasound applications.

In this short communication, a novel array structure is described to utilize 1-3 connectivity piezocrystal polymer composites in a geometrically focused array transducer. The practical challenges associated with the fabrication of the array structure and electrical interconnects to the elements are addressed. 3D printing is introduced to aid assembly. The performance of the array is demonstrated and its use with a 32-channel modular array controller is described, illustrating both beam shaping and the relocation of the focus required in focused ultrasound applications.

\section{Geodesic Structure}

The faceted spherical structure proposed in this work is drawn from the architectural design of geodesic domes. These domes comprise multiple triangles approximately equal in size, with their vertices lying on the surface of a sphere. The planes of the triangles lie physically inside the sphere but the structure approximates a sphere whilst maximizing the coverage of the aperture with the piezoelectric material.

A section of an icosahedron dome structure with dome frequency $6 \mathrm{~V}$, referring to a structure with six subdivisions of the original triangles in one icosahedron [19], was chosen for the proposed array. In total, the section requires 24 triangular flat plates positioned as shown in Fig. 1. To match a commercial piezoelectric bowl used as a reference, the target operating frequency of the array was $1.0 \mathrm{MHz}$ and its aperture was $62 \mathrm{~mm}$ across its largest diagonal, with a natural focal distance of $75 \mathrm{~mm}$, equal to the dome radius, giving $\mathrm{f} \# \approx 1.2$. Table 1 lists the four triangular geometries in this design, with their side lengths and corresponding angles. Each triangle is named for its sides taken in a clockwise direction. Five side lengths in total are needed, denoted D, F, G, H and I. Triangles HGG and HII are isosceles and triangles DFG and DGF are scalene; six of each type are required. These triangles were machined by a precision dicing saw (MicroAce 66, Loadpoint Ltd., Wiltshire, UK) with dicing position accuracy of $0.001 \mathrm{~mm}$ and theta axis resolution of $0.0005^{\circ}$.

To increase the number of array elements for more flexible control over beam forming, each triangular plate was sub-divided into four smaller triangular elements by dicing along the mid-segments of the plates to half their thickness. The number of individual transducer elements was thus increased from 24 to 96 , as shown in Fig. 1c, with only one additional step in the fabrication process. Each sub-diced element is connected to an individual signal wire at the rear and a common silver ink electrode ground with a single wire at the front. 


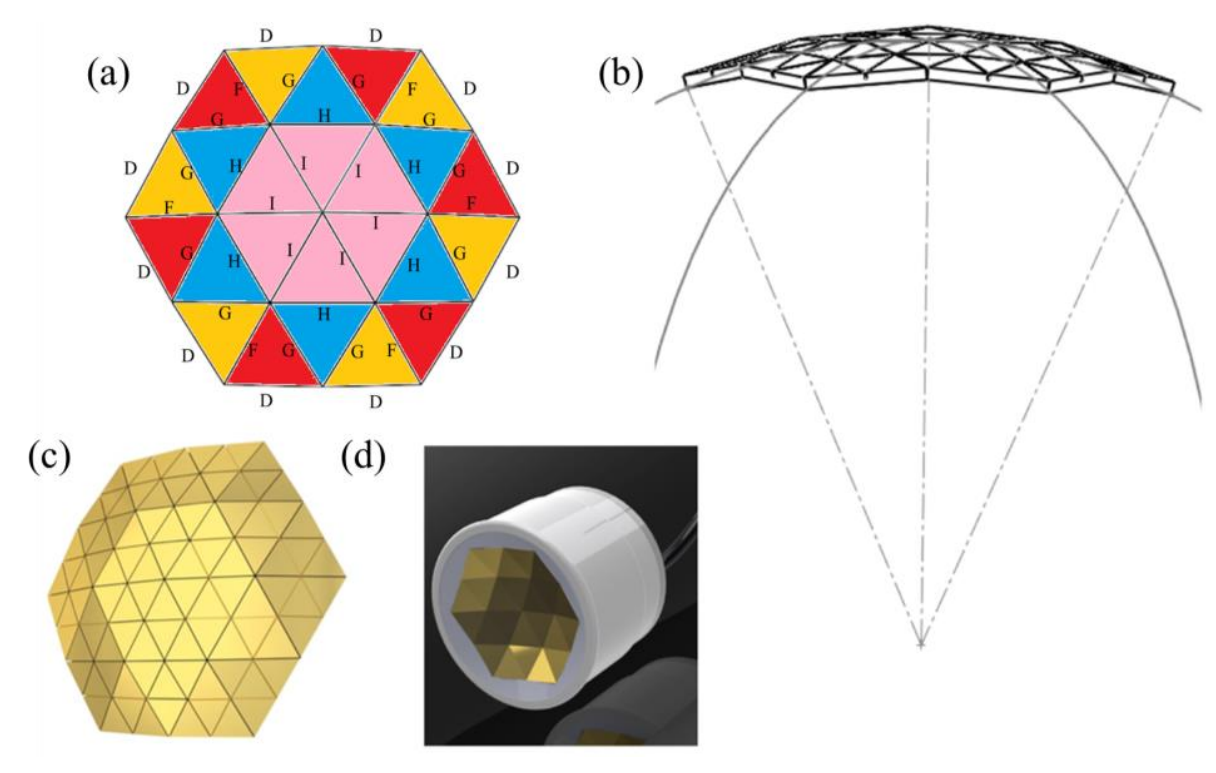

Figure 1. Illustration of geodesic array transducer. (a) Configuration of 24 triangular plates with four different shapes; (b) overall array configuration with geometrical focus; (c) rear view of the array with 96 sub-diced individual elements, (d) computer-aided design front view of the array assembly.

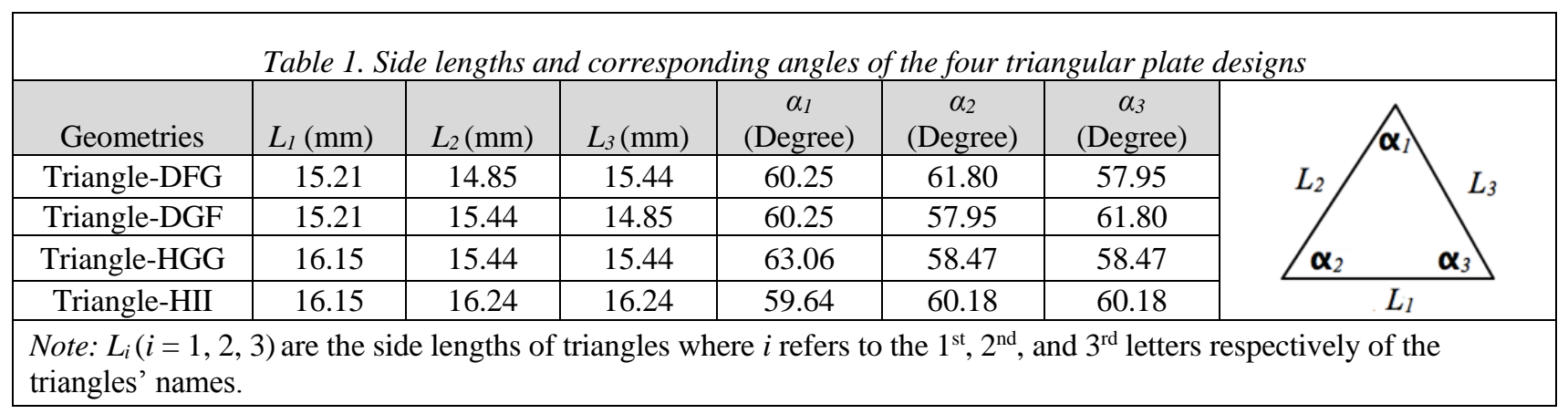

\section{Piezomaterials}

The geodesic faceted structure was realized with two different piezoelectric materials: bulk ceramic to test the fabrication process and geodesic geometry and 1-3 connectivity PMN-PT piezocrystal - epoxy composite for performance evaluation. As noted previously, 1-3 composite is necessary to take advantage of the high values of $k_{33}$ and $d_{33}$ of PMN-PT.

For initial evaluation of fabrication, a pre-electroded piezoceramic plate, thickness $2 \mathrm{~mm}$ for $1 \mathrm{MHz}$ operational frequency, (PZ26, Meggitt Sensor Systems, Kvistgaard, Denmark) was diced and separated into 24 triangular plates and then sub-diced into 96 sub-elements with $210 \mu \mathrm{m}$ kerf width and $1 \mathrm{~mm}$ depth. Similarly, another set of 24 triangular plates was diced from 1-3 connectivity PMN-29\%PT (Sinoceramic Inc., Shanghai, China) - hard polymer (Epofix, Struers A/S, Ballerup, Denmark) piezocomposite fabricated in house using the dice-and-fill method. Epofix was chosen for its low shrinkage, mechanical strength, low Poisson's ratio, and chemical resistance [20]. The piezocomposite material had a dicing kerf of $113 \mu \mathrm{m}$, a volume fraction, $V F=60 \%$, and a pillar aspect ratio (width to thickness), $P A R=0.32$. It was lapped to a 
thickness of $1.2 \pm 0.02 \mathrm{~mm}$ to achieve the $1.0 \mathrm{MHz}$ operational frequency. Electrodes were applied on both sides of the triangular piezocomposite plates using silver ink (118-09A/B, Creative Materials Incorporated, USA) cured at $65^{\circ} \mathrm{C}$ for $16 \mathrm{~h}$. One electrode face on each triangular piezocomposite plate was part-diced into four smaller triangular divisions, while the ground electrodes on the opposite faces were left intact.

\section{Array Assembly}

The process to assemble the array transducer from the machined triangular plates is illustrated in Fig. 2, with a corresponding photograph for each step.

An important aspect of this process is the placement of each triangular plate in the position needed to create the $3 \mathrm{D}$ faceted structure. The target wavelength, $\lambda \approx 1.5 \mathrm{~mm}$, indicates the need for precision much better than $1 \mathrm{~mm}$. Hence, an ABS thermoplastic mould with faceted surfaces matching the geodesic design was made by 3D printing (uPrint SE 3D Printer, Stratasys Ltd, USA), as seen in Fig. 2a and 2c. The layer resolution of the $3 \mathrm{D}$ printer was $0.254 \mathrm{~mm}$. Honey thinned in a small container in a warm water bath at $50^{\circ} \mathrm{C}$ was used to hold the plates in place, providing lower viscosity compared to other temporary adhesive options such as silicon grease and thus minimizing the layer thickness on the mould (Fig. 2b). The use of honey also allows the mould to be released and the residue to be removed with water. The triangular composite plates were placed on the mould in the arrangement indicated in Fig. 1b and secured with 5 min-curing epoxy (Araldite ${ }^{\circledR}$ Rapid, Huntsman Advanced Materials GmbH, Switzerland), (Fig.2d, e).

Because of the thermal sensitivity of PMN-PT piezocrystal, with rhombohedral to tetragonal phase transition temperature, $T_{R T} \approx 80^{\circ} \mathrm{C}$ [21], conductive silver epoxy (G3349, Agar Scientific, UK) rather than soldering was used for the electric connections. Silver ink was also discarded as an option as its viscosity is much lower than that of silver epoxy and it does not have the physical integrity to maintain its shape during curing. Since this requires $\sim 30$ mins curing time at $55^{\circ} \mathrm{C}$, custom-designed interconnect upstands, Fig. $2 \mathrm{~g}$, were made to hold the cables in place during curing. These also allowed multiple connections to be made together, increasing the assembly efficiency. Each upstand took the form of a hexahedron comprising two plastic nuts stacked together (528-132, RS Components, Northants, UK). A flexible printed circuit board (fPCB) made in house was attached around the hexahedron and alternate active and ground tracks were connected to micro co-axial cables taken from commercial devices withdrawn from service (Toshiba Medical Systems Europe, Zoetermeer, Netherlands). Each upstand supported six interconnects and the hexahedral shape allowed fan-out of the active tracks to minimize the risk of shorting. Once each upstand had been 
prepared, it was fixed to the back of the array element assembly with the 5 min-curing epoxy and the signal tracks were connected to the sub-diced triangular composites with conductive silver epoxy (Fig. 2f, g, and h). 19 upstands were required for the array: 13 centrally with six micro co-axial cables each, and six at the perimeter of the array with three micro co-axial cables each. The array assembly was then transferred into a pre-heated oven to cure the conductive silver epoxy.

Integration with the outer casing followed. A mixture of micro-balloons (K1, 3M, USA) and epoxy (Epofix, Struers A/S, Ballerup, Denmark), mass ratio 1:2, was compressed carefully onto the back of the array element assembly between the interconnect upstands, to act as a mechanical support for the array but with reduced acoustic damping compared with unfilled epoxy because of the higher attenuation and lower acoustic impedance. These parameters cannot be quantified as it is impossible to achieve penetration of sound through the filled epoxy to measure them. After the backing layer was cured, the assembly was released from the mould and the casing was sealed with a watertight rear cover (Fig.2i, j). Finally, remaining honey was removed with warm water and a silver ink common ground was applied on the front face of the array.

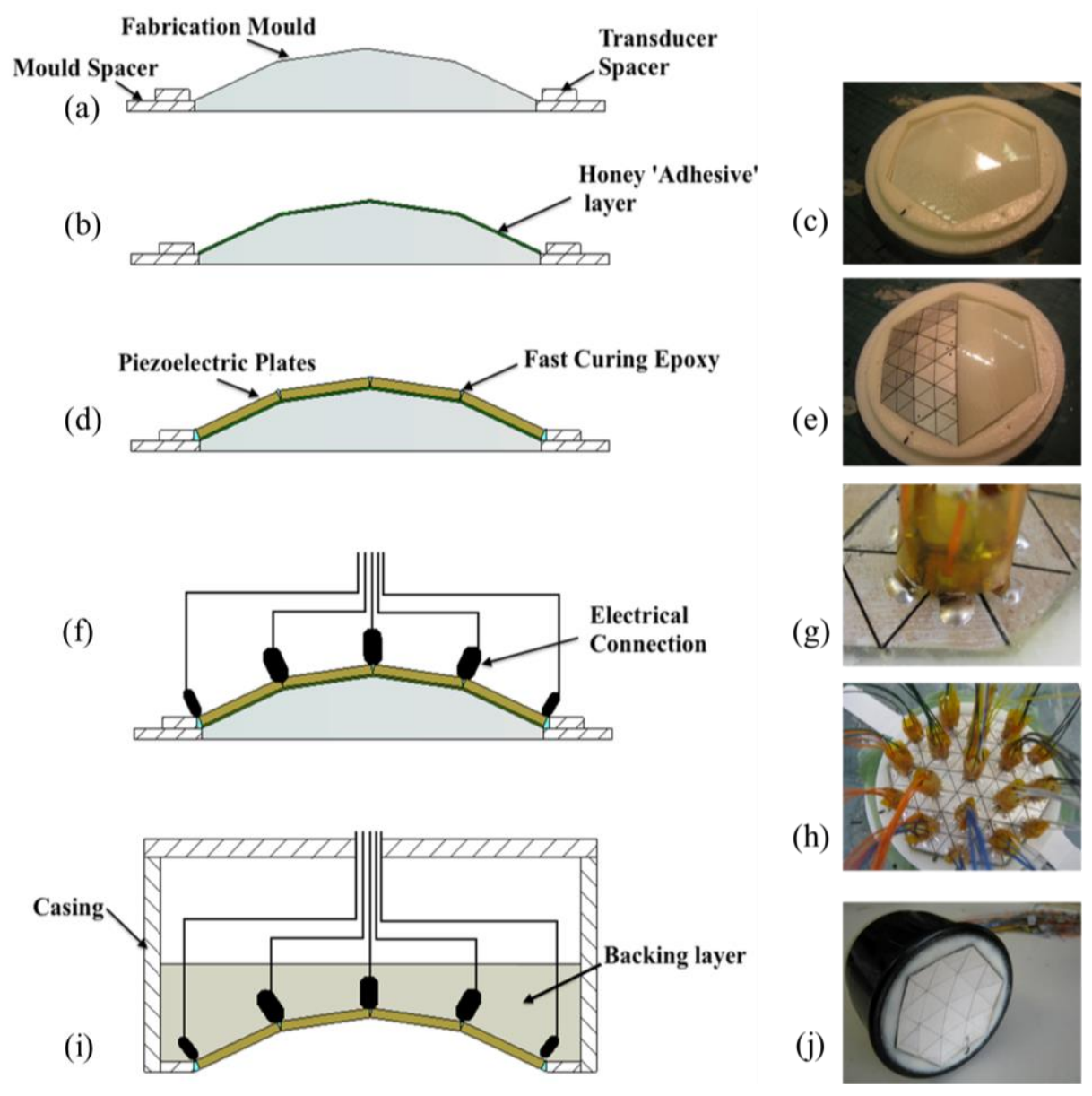

Figure 2. Assembly process for faceted bowl arrays: (a) mould with spacers, (b) with honey applied, (c) ready for the piezoelectric plates to be applied; $(d)$ with the plates in place, $(e)$ showing twelve plates, $(f)$ electrical connections added at the plate vertices; $(g)$ detail of the interconnect upstand scheme, $(h)$ shown in overview, $(i)$ with casing, backing layer and sealing plate added and $(j)$ the complete array. 


\section{Array Characterisation and Evaluation}

\subsection{Electrical Impedance Spectroscopy}

The complex electrical impedance of each of the 96 elements of the piezocrystal composite array was measured with an impedance analyzer (4395A, Agilent Technologies / Keysight, Santa Clara, CA, USA) with the results plotted in Fig. 3. All 96 elements are fully functional without open or short connections. The fundamental resonance and anti-resonance frequencies of the elements across the array, with standard deviation, are $f_{r}=1.05 \pm 0.02 \mathrm{MHz}$ and $f_{a}=1.39 \pm 0.04 \mathrm{MHz}$, respectively. The electrical impedance magnitude $|Z|=241 \pm 57 \Omega$ at the value of $f_{r}$ for each element. The reasons for the large standard deviation are three-fold: non-uniformity of piezocrystal material as purchased from two different production batches (composite velocities measured to be $3285 \mathrm{~m} / \mathrm{s}$ and $3490 \mathrm{~m} / \mathrm{s}$, respectively); variations in the individual piezocrystal composite fabrication; and the systematic size differences between the triangular plates.

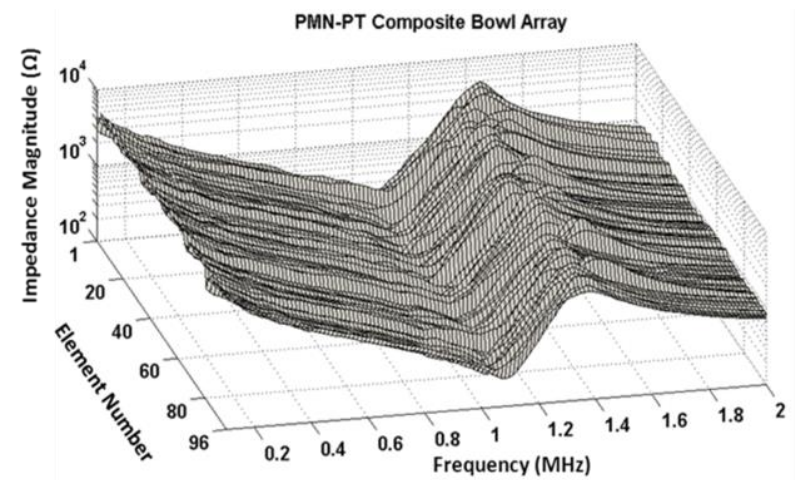

(a)

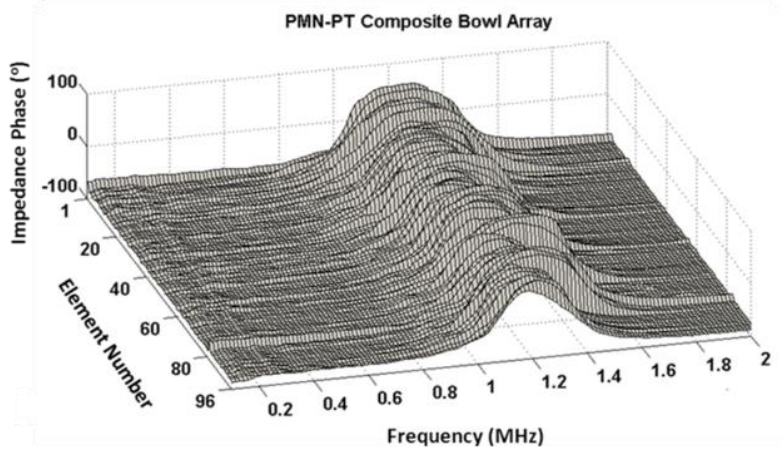

(b)

Figure 3. Complex electrical impedance (a) magnitudes and (b) phases of all 96 elements of the piezocrystal composite array

\subsection{Acoustic Pressure Field Mapping}

The arrays were connected to commercial array control electronics (FI Toolbox DSL32T, Diagnostic Sonar Ltd, Livingston, UK), customized to provide continuous wave output [22]. To match the 32 transmission channel count on the driving system, the 96 individual elements of the array were grouped into 32 channels, with sets of three adjacent elements connected together as shown in Fig. 4. 


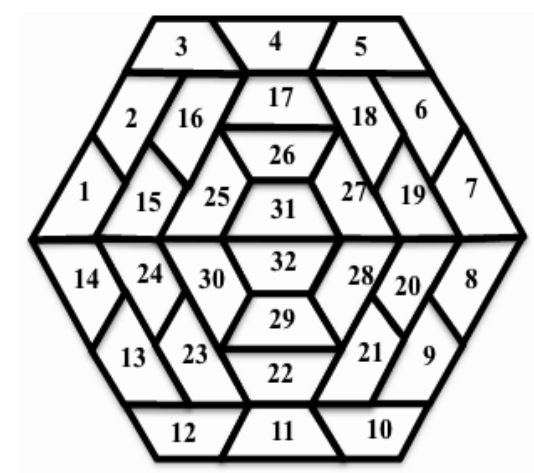

Figure 4. Sketch of the distribution of 32 channels on the geodesic array in 2D segmented annular configuration

\subsubsection{Natural focusing}

The focusing ability of the geodesic array configuration was tested initially with the bulk ceramic array, with its 32 groups of elements driven at $1.0 \mathrm{MHz}$, by mapping the beam profile over two perpendicular planes intersecting the transducer focus. To do this, the array was immersed in a tank of degassed water and a $0.5 \mathrm{~mm}$ diameter needle hydrophone (Precision Acoustics Ltd, Dorchester, UK) was attached to a 3-axis motorized scanning system to measure the acoustic intensity at $0.5 \mathrm{~mm}$ increments. A natural focus was achieved with the geodesic structure, as shown in Fig. 5. The $-3 \mathrm{~dB}$ (full width half maximum, FWHM) beam width at the focus was $2.4 \mathrm{~mm}$ and it was $21 \mathrm{~mm}$ in length. The intensities of most of secondary side lobes were below $-12 \mathrm{~dB}$ relative to the peak amplitude of the main lobe.

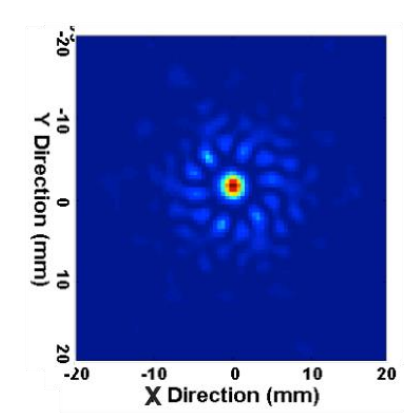

(a)

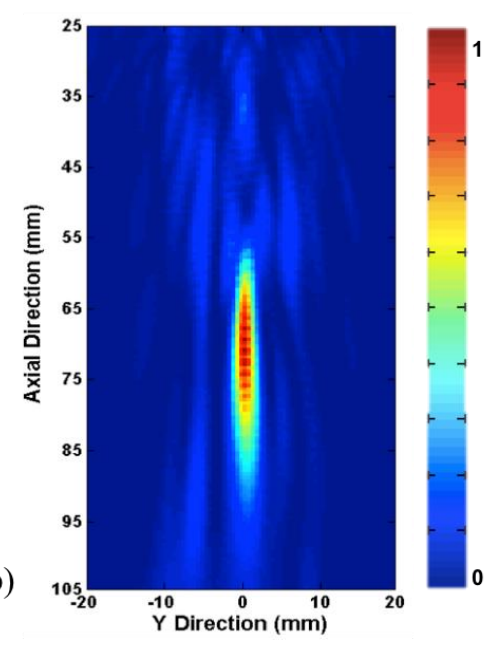

Figure 5. Normalized output acoustic intensity of the piezoceramic array in its natural focal plane (a) cross section $74.5 \mathrm{~mm}$ from the centre of the array and (b) axial scan.

\subsubsection{Electronic focusing and steering}

The piezocrystal composite array was connected to array control system setup to enable programming of phase delays for steering and focusing. A phase quantisation step of $11.25^{\circ}$ was possible, corresponding to a time delay of $31.2 \mathrm{~ns}$ at $1.0 \mathrm{MHz}$ (time period $1.0 \mu \mathrm{s}$ ). Phase correction of focusing aberrations based on hydrophone measurements was performed to allow for the increased non-uniformity of the piezocrystal 
composite elements compared with the piezoceramic. The focus of the array was found at $69 \mathrm{~mm}$ in front of the array and it was then shifted and steered axially and laterally within a volume of $10 \times 5 \times 5 \mathrm{~mm}^{3}$. Representative plots of the acoustic intensity fields are shown in Fig. 6. As an example, the -3 dB FWHM beam dimensions at the focus in Fig. $6 \mathrm{~b}$ were $1.5 \mathrm{~mm}$ in width and $15 \mathrm{~mm}$ in length. The focusing gain of the array is 30 , calculated from the square root of the ratio of the source area to the focal area. Similar to the results from the piezoceramic array, the intensities of the side lobes in the steered beam were below $-12 \mathrm{~dB}$ compared to the main lobe.
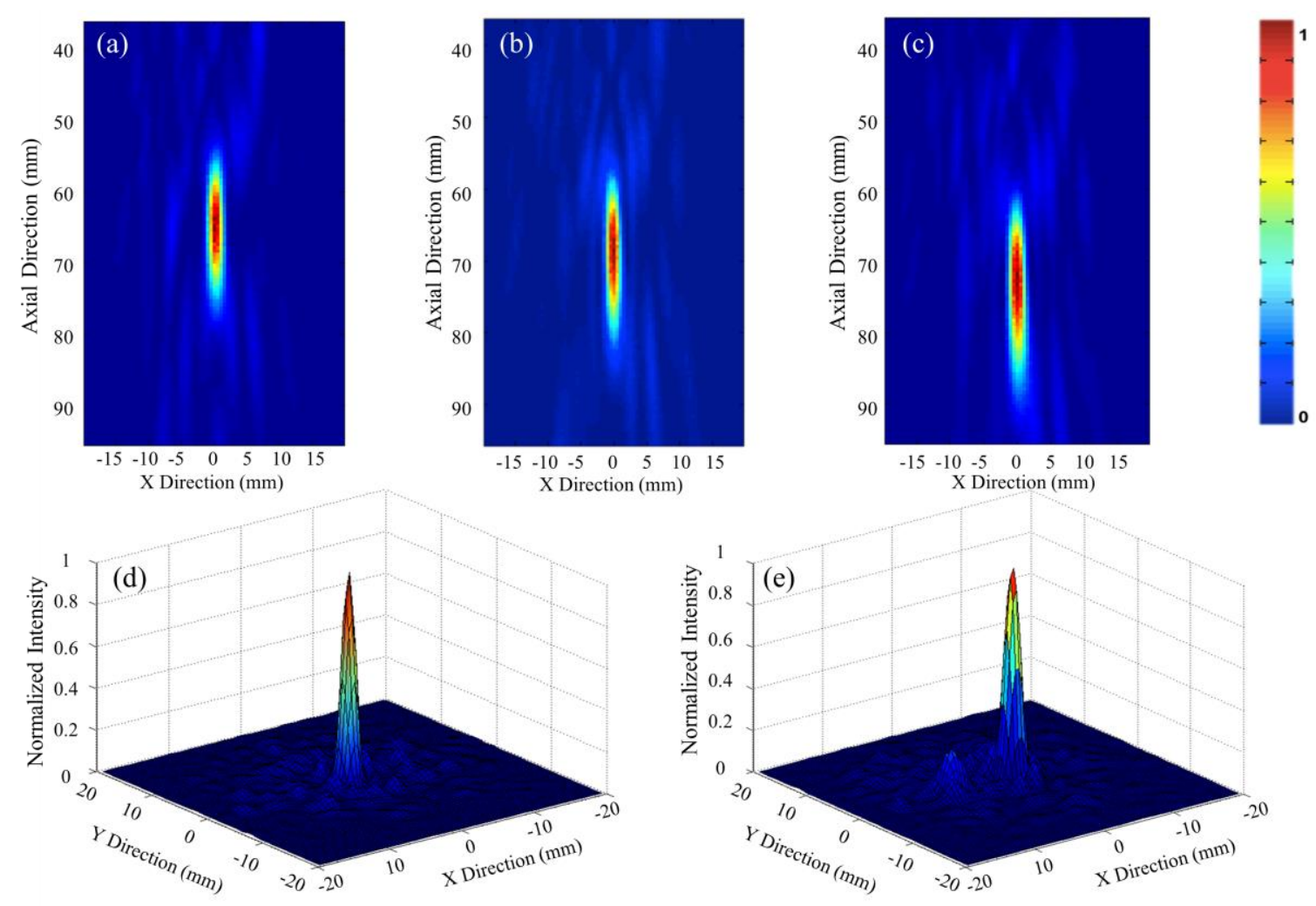

Figure 6. Normalized output acoustic intensity of the piezocrystal array with the focus set electronically to be (a) $(0,0,64) \mathrm{mm},(b)(0,0,69) \mathrm{mm},(c)(0,0,74) \mathrm{mm},(d)(0,0,74) \mathrm{mm}$, and $(e)(2.5,0,74) \mathrm{mm}$

\section{Discussion}

Although the geodesic structure in this communication has been combined with the use of relaxor-based piezocrystals, it can be applied to any material, preferentially those in which natural crystal orientation exists, to form a self-focusing bowl resembling a section of a sphere. Gen. I piezocrystal, PMN-PT, was used here because of its commercial availability, but Gens. II and III piezocrystals will be worth investigating once the materials are available and their behavior is understood [23], not least as the issue of additional DC bias at high drive levels is moot for them [21]. 
The triangles used to form the proposed geodesic structure are not standard geometries available from piezoelectric material supplies but they can be prepared easily with a dicing saw. Besides the $6 \mathrm{~V}$ icosahedron dome structure implemented here, other geodesic dome structures exist, e.g. $4 V$ and $5 V$ [19] and could be selected to match the aperture and focal length of a particular array design.

3D printing was integrated easily into our assembly process and has shown its potential to aid future ultrasonic transducer development when complex geometrical configurations are needed. The maximum build size of the printer may constrain the array size and aperture but the mould could be printed in several parts for later assembly if a larger scale transducer is needed.

Electrical interconnects for multiple elements are a challenge during array assembly, especially with complex shapes such as those discussed here. The interconnection upstands we used worked well with our array and expedited the interconnect process. Although use of flexible PCBs from array to instrumentation might further reduce the effort required for interconnection, this technique still does not offer sufficient length of track and direct cabling is thus preferred.

In the preliminary results from electronic focusing and steering, the intensities of the side lobes steering along the axis were as low as $-12 \mathrm{~dB}$ compared to the main lobe, whilst a $-9 \mathrm{~dB}$ side lobe was observed when steering the focus off the axis. This increase in sidelobe level is predictable because lateral shifting of the focus was constrained by the 32-channel configuration. Since the electronics are modular, additional channels could be configured to connect all 96 elements of the array individually, improving the focusing quality and steering range.

Defocusing and therefore the need for phase aberration correction are unavoidable because of residual aberration from the array itself and from distortion in the medium during ultrasound propagation. Considering only the device, we noted that aberration caused by assembly, associated with the accuracy of the 3D printed mould, was secondary to that caused by non-uniform elements resulting from a combination of the characteristics of the individual piezocrystal composites and the non-uniformity of the piezocrystal material [12]. The hydrophone-based phase correction we adopted gave FWHM beam diameter of approximately one wavelength, but the sidelobe level could be improved further by increasing the number of electronic channels. 


\section{Conclusions}

This communication has proposed and evaluated a novel faceted array structure inspired by geodesic dome architecture, allowing the adoption of high performance piezocrystal materials, such as PIN-PMN-PT and Mndoped PIN-PMN-PT piezocrystals, for focused ultrasound applications, since the crystal orientation in crystalline materials prevents formation into a bowl shape in the same way as piezoceramics. The details of assembly of a 96-element array have been described, based on PMN-PT piezocrystal - epoxy composite, with bulk piezoceramic providing a reference device with which good focusing was achieved. Electronic focusing was found to be necessary for the piezocrystal composite array, with satisfactory results achieved even though groups of three elements were connected. The same technique also allows steering of the focus. The array has not been tested at therapeutic levels because the PMN-PT single crystal material available for this work would require an additional DC bias field of about $400 \mathrm{~V}$, which was not available for the present work. The customized commercial electronics we used could however further extend the potential of the geodesic array for focused ultrasound applications.

\section{Acknowledgements}

In this work, Dr. Zhen Qiu was supported by the Scottish Universities Physics Alliance. The authors' contributions were made whilst they were employed by the Institute for Medical Science and Technology, University of Dundee.The authors also thank Diagnostic Sonar Ltd for providing the driving system, and Mr. Graeme Casey, University of Glasgow, and Dr. Jack Ng, Heriot-Watt University, for their assistance with this project.

\section{References}

[1] J. E. Kennedy, "High-intensity focused ultrasound in the treatment of solid tumours.," Nature Review Cancer, vol. 5, pp. 321-7, 2005.

[2] T. M. Allen, "Liposomes. Opportunities in drug delivery," Drugs, vol. 54 Suppl 4, pp. 8-14, 1997.

[3] H. M. Hertz, "Standing - wave acoustic trap for nonintrusive positioning of microparticles," Journal of Applied Physics, vol. 78, pp. 4845-4849, 1995.

[4] D. R. Daum and K. Hynynen, "A 256-element ultrasonic phased array system for the treatment of large volumes of deep seated tissue," Ultrasonics, Ferroelectrics and Frequency Control, IEEE Transactions on, vol. 46, pp. 1254-1268, 1999.

[5] E. S. Ebbini and C. A. Cain, "A spherical-section ultrasound phased array applicator for deep localized hyperthermia," Biomedical Engineering, IEEE Transactions on, vol. 38, pp. 634-643, 1991.

[6] V. Auboiroux, E. Dumont, L. Petrusca, M. Viallon, and R. Salomir, "An MR-compliant phased-array HIFU transducer with augmented steering range, dedicated to abdominal thermotherapy," Physics in Medicine and Biology, vol. 56, pp. 3563-82, 2011.

[7] Y. Qiu, H. Wang, C. E. Demore, D. A. Hughes, P. Glynne-Jones, S. Gebhardt, et al., "Acoustic devices for particle and cell manipulation and sensing," Sensors (Basel), vol. 14, pp. 14806-38, 2014.

[8] P. Sun, G. Wang, D. Wu, B. Zhu, C. Hu, C. Liu, et al., "High Frequency PMN-PT 1-3 Composite Transducer for Ultrasonic Imaging Application," Ferroelectrics, vol. 408, pp. 120-128, 2010. 
[9] M. F. Wallace, H. Mulvana, P. Marin, K. Mayne, M. P. Walsh, R. Wright, et al., "Parametric Array Design and Characterisation for Underwater Sonar and Medical Strain Imaging Applications," in Ultrasonics Symposium, 2007. IEEE, 2007, pp. 305-308.

[10] Y. Chen, K.-H. Lam, D. Zhou, Q. Yue, Y. Yu, J. Wu, et al., "High Performance Relaxor-Based Ferroelectric Single Crystals for Ultrasonic Transducer Applications," Sensors, vol. 14, p. 13730, 2014.

[11] S. Zhang, S.-M. Lee, D.-H. Kim, H.-Y. Lee, and T. R. Shrout, "Elastic, Piezoelectric, and Dielectric Properties of 0.71Pb(Mg1/3Nb2/3)O3-0.29PbTiO3 Crystals Obtained by Solid-State Crystal Growth," Journal of the American Ceramic Society, vol. 91, pp. 683-686, 2008.

[12] Z. Qiu, M. R. Sadiq, C. Demore, M. F. Parker, P. Marin, K. Mayne, et al., "Characterization of piezocrystals for practical configurations with temperature- and pressure-dependent electrical impedance spectroscopy," Ultrasonics, Ferroelectrics and Frequency Control, IEEE Transactions on, vol. 58, pp. 1793-1803, 2011.

[13] K. Yohan, A. D. Maxwell, T. L. Hall, X. Zhen, L. Kuang-Wei, and C. A. Cain, "Rapid prototyping fabrication of focused ultrasound transducers," Ultrasonics, Ferroelectrics, and Frequency Control, IEEE Transactions on, vol. 61, pp. 1559-1574, 2014.

[14] B. I. Raju, C. S. Hall, and R. Seip, "Ultrasound therapy transducers with space-filling non-periodic arrays," Ultrasonics, Ferroelectrics and Frequency Control, IEEE Transactions on, vol. 58, pp. 944-954, 2011.

[15] Q. Zhou, X. Xu, E. J. Gottlieb, L. Sun, J. M. Cannata, H. Ameri, et al., "PMN-PT single crystal, high-frequency ultrasonic needle transducers for pulsed-wave Doppler application," Ultrasonics, Ferroelectrics, and Frequency Control, IEEE Transactions on, vol. 54, pp. 668-675, 2007.

[16] K. H. Lam, Y. Chen, K. F. Cheung, and J. Y. Dai, "PMN-PT single crystal focusing transducer fabricated using a mechanical dimpling technique," Ultrasonics, vol. 52, pp. 20-24, 1// 2012.

[17] W. A. Smith, "The role of piezocomposites in ultrasonic transducers," in Ultrasonics Symposium, 1989. Proceedings., IEEE 1989, 1989, pp. 755-766 vol.2.

[18] G. Harvey, A. Gachagan, J. W. Mackersie, T. McCunnie, and R. Banks, "Flexible ultrasonic transducers incorporating piezoelectric fibres," Ultrasonics, Ferroelectrics, and Frequency Control, IEEE Transactions on, vol. 56, pp. 1999-2009, 2009.

[19] B. Carlo, "The Wooden Roofs of Leonardo and New Structural Research," Nexus Network Journal: Architecture and Mathematics, vol. 10, pp. 27-38, 2008.

[20] A. L. Bernassau, D. Hutson, C. E. Demore, and S. Cochran, "Characterization of an epoxy filler for piezocomposites compatible with microfabrication processes," IEEE Trans Ultrason Ferroelectr Freq Control, vol. 58, pp. 2743-8, Dec 2011.

[21] S. Zhang and T. R. Shrout, "Relaxor-PT single crystals: observations and developments," Ultrasonics, Ferroelectrics and Frequency Control, IEEE Transactions on, vol. 57, pp. 2138-2146, 2010.

[22] Z. Qiu, R. Habeshaw, S. Cochran, and L. Dave, "Customized modular multichannel electronics for ultrasoundmediated targeted drug delivery with a geodesic piezocrystal phased array," in Ultrasonics Symposium (IUS), 2014 IEEE International, 2014, pp. 811-814.

[23] X. Liao, Z. Qiu, T. Jiang, M. Sadiq, Z. Huang, C. Demore, et al., "Functional Piezocrystal Characterisation under Varying Conditions," Materials, vol. 8, p. 5456, 2015. 